\section{G222 DOES THE IMPLEMENTATION OF A VENTILATOR ASSOCIATED PNEUMONIA (VAP) CARE BUNDLE REDUCE INCIDENCE OF VAP IN PICU?}

CL Hill. Paediatric Intensive Care, Oxford Universities NHS Trust, Oxford, UK

10.1136/archdischild-2015-308599.215

Ventilator associated pneumonia (VAP) is pneumonia that develops $48 \mathrm{~h}$ after intubation. VAP is the second most common nosocomial infection in PICU patients and is associated with increased duration of ventilation, ICU stay, hospital stay, and cost. VAP is a preventable healthcare acquired infection and a Department of Health (1999) Saving Lives initiative. This poster aims to discover whether the introduction of a VAP care bundle (VCB) is effective at reducing VAP rates in PICU.

A search of various databases was undertaken, including MedLine, Pubmed, CINHAL, EBSCO, using relevant PICO key words. 3 paediatric research studies were found and 4 published presentations of data. Due to the limited amount of paediatric data the search was extended to include adult studies, producing 12 valid studies.

Results conclusively showed a decrease in VAP following the introduction of a VAP care bundle. Paediatric studies showed a reduction ranged from 76\%-100\% (91\% average), with 210 days -3 years VAP free with associated decreases in length of stay and cost. Adult studies found a $62 \%$ average decrease in VAP, with higher reduction in VAP associated with increased compliance.

Due to limitations of the observational designs used, various implementation strategies, and lack of consistency in VCB elements, it is difficult to determine what is responsible for the measured outcomes. However the weight of evidence showing VAP reduction with VCB use suggests a strong relationship. And although the evidence is not at the highest experimental level, it is at the highest ethically acceptable level. It should also be taking into consideration that each individual bundle component is supported by its own breadth of high level experimental evidence. Routine use of VCB is widespread in adult ICUs, and the adult and paediatric literature agree that care bundles are an effective method for reducing the incidence of ventilator associated pneumonia. Therefore in absence of any contradictory data, care bundles should be adopted in paediatric intensive care practice. Further research is required to examine individual elements of the paediatric VCB; but with a synchronised team approach, $\mathrm{VCB}$ can result in continued reductions in VAP, length of stay, and costs.

\section{G223 IMPLEMENTING AND EVALUATING MODELS OF FAMILY CENTRED CARE OR CHILDREN WITH LONG TERM CONDITIONS - WHERE NEXT?}

1J Smith, ${ }^{2} \mathrm{G}$ Fenton, ${ }^{3} \mathrm{C}$ Coyne, ${ }^{4} \mathrm{~V}$ Swallow. ${ }^{1}$ School of Human and Health Sciences, University of Huddersfield, Huddersfield, UK; ${ }^{2}$ School of Nursing, Midwifery and Social Work, University of Salford, Salford, UK; ${ }^{3}$ School of Nursing and Midwifery, Trinity College Dublin, Dublin, Ireland; ${ }^{4}$ School of Nursing, Midwifery and Social Work, University of Manchester, Manchester, UK

\subsection{6/archdischild-2015-308599.216}

Background and purpose Effective collaboration between nurses and families is fundamental to supporting children with longterm conditions and their families. The family-centred care model is widely espoused as an effective framework that facilitates parents' involvement in their child's care (Smith et al,
2015). The implementation and evaluation of this model deserves further scrutiny. This paper presents two complementary critical evaluations of family-centred care research, particularly in the context of childhood long-term conditions.

Methods A scoping review of MEDLINE, CINAHL, PSYCINFO databases from 2000 to 2014 was undertaken to identify instruments that measure family family-centred care. A complementary systematic literature search of Scopus, CINAHL, BNI databases was undertaken to identify research on the implementation family-centred care in practice.

Results Fifteen studies were identified that had used measures to evaluated family-centred care but measures were often poorly defined and generally measured satisfaction rather than collaborative practice associated with family-centred care. A lack of clarity surrounds the reliability and validity of some instruments used to measure family centred care. The validated Measure of Processes of Care (MPOC) was the most frequently measure used to evaluate family-centred care.

Twenty studies were reviewed to identify the key antecedents of family-centred care which included: 'unclear roles and boundaries'; 'entrenched professional practices and attitudes towards working with families'; 'lack of organisational or managerial guidelines or policies specifically aimed at supporting the implementation of family-centred care'. The key attributes associated with family centred care were: 'valuing parents knowledge and experiences', 'supporting parents in their role as care giver'; 'incorporating parents' expertise into clinical and psychosocial care', are particularly salient when empowering and supporting children, young people and families in the management of longterm conditions in children.

Conclusion Parents develop considerable expertise in managing their child's long-term condition but they perceive their expertise is not always valued and the quality of parent-professional interactions was variable (Smith, et al. 2013). Promoting and facilitating the child, young people and family's involvement in the care of children with long-term conditions requires nurses to practice family centred care (Shields et al 2012), yet tools to measure implementation and evaluation studies are limited.

\section{G224 A SYSTEMATIC REVIEW TO DETERMINE IF UNDERNUTRITION IS PROGNOSTIC OF INFECTION COMPLICATIONS IN CHILDREN FOLLOWING SURGERY}

${ }^{1,2} \mathrm{R}$ Hill, ${ }^{3} \mathrm{~S}$ Paulus, ${ }^{4} \mathrm{MP}$ Dey, ${ }^{2} \mathrm{MA}$ Hurley, ${ }^{2} \mathrm{~B}$ Carter. ${ }^{1}$ Department of Cardiac Surgery, Alder Hey Children's NHS Trust, Liverpool, UK; ${ }^{2}$ School of Health, University of Central Lancashire, Preston, UK; ${ }^{3}$ Department of Immunology and Infectious Disease, Alder Hey Children's NHS Trust, Liverpool, UK; ${ }^{4}$ School of Medicine and Dentistry, University of Central Lancashire, Preston, UK

\subsection{6/archdischild-2015-308599.217}

Background Hospitalisation has a major impact on the nutritional status of children ${ }^{1}$. Although incidence of undernutrition has been reported, fewer studies have investigated its effect on patient outcome following paediatric surgery.

Review methods

Key electronic bibliographic and research databases were searched. Inclusion criteria were studies in children $<18$ years evaluating preoperative nutritional status and reporting postoperative infection complications. Quality assessment was performed using Newcastle-Ottawa Scale ${ }^{2}$ for cohort and case-control studies. Appraisal and data extraction were performed independently by two reviewers. Effect estimates and 95\% confidence intervals 
were extracted or calculated using Chi Square or Fisher's Exact tests as appropriate.

Results Two reviewers screened a total of 1108 references. Ten cohort and two case-control studies using a plethora of nutritional assessments were included in the review. Postoperative infection complications reported were either combined or individual e.g. wound infection. Quality of the evidence was judged as low in the majority of studies, with two of moderate and two of very low quality. Direct comparison between studies was not possible due to clinical and diagnostic heterogeneity. Direction of effect on univariate analysis was suggestive of a relationship between undernutrition and postoperative infection complications.

Conclusion The lack of a consistently applied method of nutritional assessment, combined with small sample sizes, makes it difficult to draw strong conclusions. There is tentative low quality evidence suggesting undernutrition may be predictive of combined infection complications following surgery in children, but insufficient evidence to determine if this relationship persists when considering specific infection complications. Larger studies, using gold-standard nutritional assessment, and designed to investigate undernutrition with outcome are warranted to investigate this relationship further.

\section{REFERENCES}

1 Pichler J, Hill SM, Shaw V, Lucas A. Prevalence of undernutrition during hospitalisation in a children's hospital: what happens during admission? Eur J Clin Nutr 2014; 68:730-735

2 Wells GA, Shea B, O'Connell D, et al. The Newcastle-Ottawa Scale (NOS) for assessing the quality of nonrandomised studies in meta-analyses. http://www.ohri. ca/programs/clinical_epidemiology/oxford.asp, 2011

\section{G225(P) DOES WEARING A UNIFORM MATTER? THE IMPACT OF RESEARCH NURSES WEARING A UNIFORM WHIST SEEKING PARENTAL CONSENT TO RESEARCH TRIALS}

J Spry, N Holdback. Paediatric Intensive Care, Birmingham Children's Hospital, Birmingham, UK

\subsection{6/archdischild-2015-308599.218}

Background and aims In a regional Paediatric Intensive Care Unit (PICU) the research team have been recruiting to three multi-centre randomised controlled trials. The team were interested to explore parent's preference for the research nurses wearing a uniform whilst recruiting to these trials.

Method 62 questionnaires were given out to non-research PICU staff (nursing, administrative and medical staff). Multiple choice questions asked what they would prefer to see research nurses wearing when approaching parents about research. 24 questionnaires were given out to parents who had been approached about research trial participation in PICU. Multiple choice questions asked whether they would prefer to be approached for consent by a research nurse wearing a clinical nurse uniform, alternative uniform or their own clothes (pictorial representations given).

Results Parental questionnaire return rate was 67\%. Responding parents favoured $(62 \%)$ a uniform with reasons given including ease of identification, trust and professionalism. From the options provided, only $6 \%$ chose the 'own clothes' option. $32 \%$ reported no preference.

$31 \%$ of parents felt that what the nurse was wearing was likely to have influenced their decision to give their consent, no information was given as to whether this would be in a positive or negative way.

Staff questionnaire return rate was 73\%. 67\% preferred a uniform, 22\% an 'own clothes' option and 11\% reporting no preference.

Conclusion The results display a preference towards research nurses wearing a uniform, however there is no agreement as to what that uniform should be.

Further research would be needed to conclude whether the wearing of a uniform influences parental decision to consent for their child to participate in research trials and before any recommendations can be made for practice.

\section{G226(P) CIRCLE: ONE WAY OF DEVELOPING INTERNATIONAL RESEARCH PRACTICE}

1,2,3 B Carter, ${ }^{3,4} \mathrm{~K}$ Ford, ${ }^{5} \mathrm{~A}$ Dickinson, ${ }^{2,6} \mathrm{~L}$ Bray, ${ }^{5,7} \mathrm{~T}$ Water, ${ }^{1,2} \mathrm{~J}$ Arnott. ${ }^{1}$ School of Health, University of Central Lancashire, UK; ${ }^{2}$ Children's Nursing Research Unit, Alder Hey Children's NHSFT, UK; ${ }^{3}$ School of Health Sciences, University of Tasmania, Australia; ${ }^{4}$ Practice Development Unit, Royal Hobart Hospital, Tasmania; ${ }^{5}$ Centre for Child Health Research, AUT University, New Zealand; ${ }^{6}$ Faculty of Health and Social Care, Edge Hill University, UK; ${ }^{7}$ Research Centre, Starship Children's Health, New Zealand

\subsection{6/archdischild-2015-308599.219}

Aim Researchers and academics face many research imperatives ranging from generating income, undertaking high quality research, writing 5 star papers, developing a national and international profile through to undertaking international research. None of this is easy. This presentation aims to explore and reflect on the process and successes achieved so far by the founding members of a community of research practice - Circle. Methods Although perhaps not an obvious academic setting, Circle was developed whilst walking. Many good ideas emerge when friends and colleagues are given the chance to meet, talk, breathe and reflect. We walked and talked across parts of Tasmania, New Zealand and the UK; these walks were separated by months, geography and competing commitments. The initial three walker-researchers co-opted colleagues also working with children and families into a group that would eventually become Circle. Together we mapped our research interests and the research priorities of our home institutions and countries and started to identify research topics which would mean that our international efforts would meet various performance objectives. We discussed funding opportunities and thought about creative ways of building sound international research based on modest resources. We identified ways in which we could capitalise on each others' strengths and expertise. We also identified ways of building strong co-authorship based on our joint research and common interests.

Results From tentative steps we have created a robust and growing Circle of researchers who are working together, providing advice, support and mentorship. Measureable achievements include publication of a textbook on nursing children, co-authored papers and co-presented papers, international supervision of research students, development of a website and completion of an international photo-elicitation study.

Conclusion Opportunities exist for international work but these require innovative thinking. Sharing ideas and supporting each other has widened and deepened our thinking about research and practice and created a sense of camaraderie. And we continue to walk together whenever we get a chance. 